\title{
Prevalence and risk factors of chronic rhinosinusitis in South Korea according to diagnostic criteria*
}

\author{
Ji Hoon Kim, Changil Cho, Eun Jung Lee, Yun Suk Suh, Byeong II Choi, \\ Kyung-Su Kim
}

Department Otorhinolaryngology, Yonsei University College of Medicine, Seoul, Korea
Rhinology 54: 329-335, 2016

DOI:10.4193/Rhino15.157

*Received for publication:

June 9, 2015

Accepted: May 15, 2016

\begin{abstract}
Background: We aimed to compare the prevalence and risk factors of chronic rhinosinusitis (CRS) using two different diagnostic criteria with the same statistical data from the Korean National Health and Nutrition Examination Survey in 2009.

Methods: 'Symptom-based CRS' was defined as CRS diagnosed by questionnaires related to nasal symptoms. 'Endoscopy-based CRS' was defined based on endoscopic findings and nasal symptoms of symptom-based CRS.

Results: The overall prevalence of CRS based on the different diagnostic criteria was as follows: symptom-based CRS was $10.78 \%$ (797 of 7,394) and endoscopy-based CRS was $1.20 \%$ (88 of 7,343). Comparing symptom-based CRS to endoscopy-based CRS showed slight agreement (kappa $=0.183(0.150-0.216,95 \%$ confidence interval)). Allergic rhinitis was identified as a common risk factor for CRS based on the two diagnostic criteria.

Conclusions: The prevalence and risk factors of CRS were quite different from each other according to the different criteria, even in the same population. Therefore, it would be important to consider what specific diagnostic criteria have been adopted in the studies comparing the prevalence of CRS.
\end{abstract}

Key words: sinusitis, prevalence, diagnosis, rhinitis, allergic

\section{Introduction}

Chronic rhinosinusitis (CRS) is one of the most prevalent chronic diseases worldwide, characterized by inflammation of the paranasal sinus mucosa for 12 consecutive weeks or more, which causes significant direct medical costs ${ }^{(1-3)}$. CRS has substantial negative effects on quality of life including bodily pain, general health, vitality, and social functioning. Comparisons with other chronic diseases such as congestive heart failure, angina, chronic obstructive pulmonary disease, and back pain have revealed significantly lower scores for bodily pain and social functioning among CRS patients ${ }^{(2,4)}$. In addition, CRS is considered to be an important hidden factor that exacerbates asthma and other chronic lung diseases ${ }^{(4)}$. Considering that CRS is one of the most common chronic inflammatory diseases with a significant socioeconomic burden, more accurate data about the prevalence and risk factors of CRS should be obtained through a population-based study. Most studies on CRS have focused on diagnosis and treatment, while relatively few studies have evalu- ated the epidemiology of the disease. In previous epidemiologic studies, the prevalence of CRS was shown to vary widely from country to country and according to survey time ${ }^{(5)}$. The reason for this variation could be differences in the diagnostic criteria of each study in addition to sociodemographic factors. Recently, diagnostic criteria for an epidemiologic study were proposed by the European Position Paper on Rhinosinusitis and Nasal Polyps (EP3OS) $2012^{(6-9)}$. In some epidemiologic studies, CRS has been diagnosed based on answers to symptom-based questionnaires on specific nasal symptoms or a medical history indicating a subject has CRS ${ }^{(10,11)}$. In addition, CRS has been diagnosed by endoscopic examination of the nasal cavity with specific nasal symptoms ${ }^{(12,13)}$. Therefore, we sought to compare the prevalence and risk factors of CRS as diagnosed by two different diagnostic criteria, symptom-based and endoscopy-based, using the same statistical data from the 2009 Korean National Health and Nutrition Examination Survey (KNHANES). 


\section{Materials and methods}

Subjects and survey methods

KNHANES is a complex survey because the data was not obtained using a simple random sample, but rather a complex, multistaged, probability sampling design was used to select the participants as a whole. A total of 12,722 individuals from 4,000 households participated in the KNHANES performed from January to December 2009, and these randomly selected participants represented the general population of South Korea. Within the visiting survey team, a resident of the Korean Society of Otorhinolaryngology-Head \& Neck Surgery was included. At least second year residents of the Korean Society of Otorhinolaryngology-Head \& Neck Surgery performed the endoscopic examinations. Before the examination, the Korean Society of Otorhinolaryngology-Head \& Neck Surgery educated the participating residents about the protocol and the examination methods for standardization of the endoscopic examination. Baseline information for each population was obtained using a structured questionnaire administered by trained interviewers. After the baseline survey, the residents asked more specific questions on the questionnaire concerning the following nasal symptoms: mucopurulent discharge, nasal obstruction/congestion, facial pain/pressure, and dysosmia lasting longer than 3 months. At the same time, the residents performed a physical examination on individuals 12 years or older using a $4-\mathrm{mm}, 0^{\circ}$ rigid nasal endoscope in a well-equipped mobile medical examination bus. Nasal endoscopic examination was performed 5 minutes after applying a topical vasoconstrictor $(0.5 \%$ neosynephrine : distilled water $=1: 1$ )

\section{Definition of CRS based on different diagnostic criteria} This study has been reviewed and approved by Gangnam Severance Hospital, Institutional Review Board (3-2013-0223) with the Treaty of Helsinki. We assessed the prevalence and risk factors for CRS based on two diagnostic criteria. 'Symptombased CRS' was defined as a diagnosis based on responses to questionnaires assessing specific nasal symptoms. The questionnaires consisted of symptoms indicating sinusitis such as anterior/posterior nasal drip, nasal obstruction, facial pain, anosmia or hyposmia. Symptom-based CRS was defined on the EP3OS 2012 diagnostic criteria for epidemiologic studies (Table 1) ${ }^{(9)}$. 'Endoscopy-based CRS' was defined as a diagnosis based on endoscopic findings of mucopurulent rhinorrhea in the middle meatus or nasal polyps along with nasal symptoms meeting the definition criteria of symptom-based CRS.

\section{Possible risk factors}

The statistical association between CRS and risk factors was analyzed. The possible risk factors comprised clinically relevant sociodemographic factors and personal medical factors. These factors were based on previous epidemiologic studies regarding
CRS, and we chose 11 variables ${ }^{(6,11-13)}$. Details included sex, age, smoking, education, stress, influenza vaccination, hypertension, diabetes mellitus, bronchial asthma, allergic rhinitis and nasal septum deviation (Table 2). Age was set as a numerical variable, and the other factors were set as categorical variables. All variables except nasal septal deviation were defined based on the answers to the questionnaire. As for smoking, only current smokers were categorized as smokers and the others were categorized as nonsmokers. Concerning education, those who graduated from university were categorized into the high education group, while the others were categorized into the low level group. According to subjective feelings about stress in daily life, stress was categorized as high or low. A positive value for influenza vaccination was defined by an affirmative answer to questions such as "Have you received an influenza vaccination during the past year?" Positive values for hypertension, diabetes mellitus, bronchial asthma, and allergic rhinitis were defined by an affirmative answer to questions such as "Have you ever been diagnosed with that specific disease by a doctor?" 'Doctor-based' diagnosis of allergy and asthma was based on the subjects' history of diagnosis with allergy and asthma as recorded in KNHANES. The diagnosis of allergy and asthma was made by general physicians or specialists. Nasal septum deviation was confirmed on physical examination by an otolaryngologist during the survey. Examination of nasal septal deviation was also done by a nasal endoscope after nasal decongestion. A deviated nasal septum was defined as the presence of an asymmetric displacement to one or both sides of the nasal cavity, leading to a narrowing of the nasal cavity.

\section{Statistical analysis}

To estimate the entire non-institutionalized Korean population from the survey sample, the KNHANES sampling weight variables, masked variance primary sampling unit and stratum variables were used. Survey sample weights were used in all the analyses. Missing data were considered to be missing completely at random. The data were analyzed with SAS software (version 9.2, SAS Institute Inc., Cary, NC, USA) in order to incorporate sample weights and adjust the analysis for the complex sample design of the survey. The prevalence of CRS based on different diagnostic criteria was estimated, and the results were compared with each other using Cohen's kappa statistics to evaluate the strength of agreement. Sensitivity, specificity, positive predictive values (PPV), and negative predictive values (NPV) were calculated comparing symptom-based CRS with endoscopybased CRS. McNemar's test was used to compare the differences of each sensitivity, specificity, PPV, and NPV. Multiple logistic regression analysis was performed with 11 variables to calculate adjusted odds ratios (OR) and their $95 \%$ confidence intervals (Cl). The analysis was performed on each CRS diagnostic criteria set. A p-value $<0.05$ was considered statistically significant. 
Table 1. Definition of CRS based on different diagnostic criteria.

Symptom-based CRS
Inflammation of the nose and the paranasal sinuses characterized
by two or more symptoms, one of which should be either
nasal blockage / obstruction / congestion or nasal discharge (ante-
rior/posterior nasal drip)
$\quad \pm$ facial pain/pressure
$\quad \pm$ reduction or loss of smell
Duration of symptoms > 12 weeks
Endoscopy-based CRS

Endoscopic findings of mucopurulent rhinorrhea or nasal polyps and

Nasal symptoms meeting the criteria of 'symptom-based CRS'

CRS = chronic rhinosinusitis.

\section{Results}

Prevalence of CRS based on different diagnostic criteria Among 8,926 individuals that were 12 years or older and underwent clinical examination, participants who had any missing data values necessary for diagnosis of CRS were excluded, 7,893 individuals were finally selected for inclusion in this study. Among the 7,893 individuals, however, 499 individuals were excluded if they failed to complete key demographic data which allowed the diagnosis of chronic rhinosinusitis to be made. Also, 51 individuals did not undergo endoscopic examination due to pain or susceptibility. Thus, data from 7,343 individuals who underwent endoscopic examination were finally included for analysis. Among these 7,343 individuals, 3,458 (43.8\%) were men, and 4,435 (56.2\%) were women. The mean age of the individuals was $39.06( \pm 22.58)$ years. Although KNHANES included individuals 6 years or older, the rhinologic examination was only performed on those 12 years or older due to safety concerns with using a vasoconstriction during the endoscopic examination. The number of individuals of ages 6 to 12 years was 921 (9.35\%), while the number of individuals over 12 years of age comprised 8,926 (90.65\%) of the 12,722 individuals in the entire study population.

The overall prevalence of CRS based on different diagnostic criteria was as follows: symptom-based CRS was 10.78\% (797 of 7,394) and endoscopy-based CRS was $1.20 \%$ (88 of 7,343). Comparing symptom-based CRS to endoscopy-based CRS showed slight agreement (kappa $=0.183(0.150-0.216,95 \%$ confidence interval)) in assessing the prevalence of CRS. Because endoscopy-based CRS contained both endoscopic findings and symptom-based criteria, it was regarded as the standard of CRS diagnosis. Compared to endoscopy-based CRS, the sensitivity of symptom-based CRS was 1 , and the specificity was 0.903 , PPV
Table 2. Definition of factors applied in the analysis.

\begin{tabular}{|c|c|}
\hline Variables & Definitions \\
\hline Smoking & $\begin{array}{l}(-) \text { : former smokers or nonsmokers } \\
(+) \text { : current smokers }\end{array}$ \\
\hline Education & $\begin{array}{l}\text { - Low: graduated middle \& high school } \\
\text { •High: graduated university }\end{array}$ \\
\hline Stress & $\begin{array}{l}\text { Light or heavy: subjective feelings of stress } \\
\text { in daily life }\end{array}$ \\
\hline Influenza vaccination & $\begin{array}{l}(-) \text { or }(+) \text { : history of influenza vaccination in } \\
\text { the last year }\end{array}$ \\
\hline Hypertension & $\begin{array}{l}(-) \text { or }(+) \text { : history of hypertension diag- } \\
\text { nosed by a doctor }\end{array}$ \\
\hline Diabetes mellitus & $\begin{array}{l}\cdot(-) \text { or }(+) \text { : history of diabetes mellitus diag- } \\
\text { nosed by a doctor }\end{array}$ \\
\hline Bronchial asthma & $\begin{array}{l}\cdot(-) \text { or }(+) \text { : history of bronchial asthma } \\
\text { diagnosed by a doctor }\end{array}$ \\
\hline Allergic rhinitis & $\begin{array}{l}\cdot(-) \text { or }(+) \text { : history of allergic rhinitis diag- } \\
\text { nosed by a doctor }\end{array}$ \\
\hline Nasal septum deviation & $\begin{array}{l}\text { •(-) or (+): nasal septum deviation confir- } \\
\text { med by an otolaryngologist during the } \\
\text { survey }\end{array}$ \\
\hline
\end{tabular}

was 0.110 , and NPV was 1.

Risk factors for CRS based on different diagnostic criteria Significant risk factors for CRS based on different diagnostic criteria were as follows: symptom-based CRS - sex, age, stress level, asthma, nasal septal deviation, allergic rhinitis (Table 3); endoscopy-based CRS - sex, age, education level, diabetes mellitus, allergic rhinitis (Table 4). Allergic rhinitis was identified as a common risk factor for CRS based on the two diagnostic criteria.

\section{Discussion}

Diagnostic criteria for symptom-based CRS are useful for largescale studies because of convenience in the survey. Nevertheless, there are some difficulties in distinguishing CRS from other diseases that cause similar nasal symptoms like allergic rhinitis, chronic rhinitis, septal deviation and the common cold. In this study, oedema of the middle meatus was not included as a positive finding, as nasal mucosal shrinkage was performed for better visualization of the nasal cavity and to reduce pain during examination. Furthermore, Tomassen et al. mentioned that endoscopic findings, such as edema or redness, can also be present in chronic rhinosinusitis and allergic rhinitis, this may account for a high proportion of positive endoscopic findings in CRS-negative allergic rhinitis patients ${ }^{(14)}$. Moreover, the Global allergy and asthma European network (GA2LEN) study included positive endoscopic findings as edema and showed a significant association between symptom-based CRS and positive endoscopic findings in non-allergic subjects only ${ }^{(14)}$. Therefore, our di- 
Table 3. Multiple logistic regression analysis of risk factors of symptom-based CRS.

\begin{tabular}{|c|c|c|c|c|c|c|}
\hline Variables & No. & CRS & Prevalence (\%) & Adjusted OR & $95 \% \mathrm{Cl}$ & p value \\
\hline \multicolumn{7}{|l|}{ Sex } \\
\hline Female & 4200 & 411 & 9.79 & 1 & & \\
\hline Male & 3194 & 386 & 12.09 & 1.351 & $1.108-1,648$ & 0.0030 \\
\hline Age $(\geq 19)$ & 7394 & 797 & 10.78 & 1.007 & $1.001-1.014$ & 0.0339 \\
\hline \multicolumn{7}{|l|}{ Smoking } \\
\hline$(-)$ & 5730 & 600 & 10.47 & 1 & & \\
\hline$(+)$ & 1641 & 195 & 11.88 & 1.041 & $0.855-1.266$ & 0.6917 \\
\hline \multicolumn{7}{|l|}{ Education } \\
\hline High level & 1907 & 197 & 10.33 & 1 & & \\
\hline Low level & 5428 & 593 & 10.92 & 1.162 & $0.940-1.436$ & 0.1664 \\
\hline \multicolumn{7}{|l|}{ Stress } \\
\hline Light & 5164 & 525 & 10.17 & 1 & & \\
\hline Heavy & 2180 & 267 & 12.25 & 1.247 & $1.009-1.541$ & 0.0413 \\
\hline \multicolumn{7}{|c|}{ Influenza vaccination } \\
\hline$(-)$ & 4834 & 493 & 10.20 & 1 & & \\
\hline$(+)$ & 2537 & 302 & 11.90 & 1.162 & $0.949-1.421$ & 0.1459 \\
\hline \multicolumn{7}{|l|}{ Hypertension } \\
\hline$(-)$ & 5893 & 633 & 10.74 & 1 & & \\
\hline$(+)$ & 1500 & 163 & 10.87 & 0.840 & $0.658-1.072$ & 0.1602 \\
\hline \multicolumn{7}{|l|}{ Diabetes mellitus } \\
\hline$(-)$ & 6823 & 739 & 10.83 & 1 & & \\
\hline$(+)$ & 570 & 57 & 10.00 & 0.721 & $0.506-1.028$ & 0.0707 \\
\hline \multicolumn{7}{|l|}{ Bronchial asthma } \\
\hline$(-)$ & 7145 & 741 & 10.37 & 1 & & \\
\hline$(+)$ & 226 & 54 & 23.89 & 2.678 & $1.661-4.319$ & $<0.0001$ \\
\hline \multicolumn{7}{|l|}{ Allergic rhinitis } \\
\hline$(-)$ & 6631 & 595 & 8.97 & 1 & & \\
\hline$(+)$ & 740 & 200 & 27.03 & 3.906 & $3.163-4.823$ & $<0.0001$ \\
\hline \multicolumn{7}{|c|}{ Nasal septum deviation } \\
\hline$(-)$ & 4206 & 399 & 9.49 & 1 & & \\
\hline$(+)$ & 3127 & 389 & 12.44 & 1.340 & $1.115-1.611$ & $0.0018^{*}$ \\
\hline
\end{tabular}

$\mathrm{CRS}=$ chronic rhinosinusitis; $\mathrm{OR}=$ odds ratio; $\mathrm{Cl}=$ confidence interval.

agnostic criteria of 'endoscopy-based CRS' would be appropriate to control for the effect of other diseases, such as allergic rhinitis, although underestimation of the prevalence of 'endoscopybased CRS' may potentially be an important limitation of this study. In past epidemiologic studies, the reported prevalence of CRS varied widely from 1 to $16 \%$ depending on the country surveyed ${ }^{(5)}$. In addition, these studies have many differences in their criteria and population. Even in the same country, the prevalence differed depending on survey time and criteria. In Korea, three epidemiological studies were reported using a nationwide survey: one in 1996 by Min et al., one in 2010 by Cho et al., and one in 2011 by Kim et al ${ }^{(12,13,15)}$. In these studies, CRS was diagnosed by questionnaires on nasal symptoms along with positive endoscopic findings. The definition of CRS in Cho's and 
Table 4. Multiple logistic regression analysis of risk factors of endoscopy-based CRS.

\begin{tabular}{|c|c|c|c|c|c|c|}
\hline Variables & No. & CRS & Prevalence (\%) & Adjusted OR & $95 \% \mathrm{CI}$ & $p$ value \\
\hline \multicolumn{7}{|l|}{ Sex } \\
\hline Female & 4167 & 34 & 0.82 & 1 & & \\
\hline Male & 3176 & 54 & 1.70 & 2.522 & $1.390-4.573$ & 0.0023 \\
\hline Age $(\geq 19)$ & 7343 & 88 & 1.20 & 1.023 & $1.003-1.043$ & 0.0221 \\
\hline \multicolumn{7}{|l|}{ Smoking } \\
\hline$(-)$ & 5688 & 59 & 1.04 & 1 & & \\
\hline$(+)$ & 1633 & 28 & 1.71 & 1.174 & $0.632-2.179$ & 0.6122 \\
\hline \multicolumn{7}{|l|}{ Education } \\
\hline High level & 1892 & 12 & 0.63 & 1 & & \\
\hline Low level & 5393 & 74 & 1.37 & 2.525 & $1.200-5.310$ & 0.0146 \\
\hline \multicolumn{7}{|l|}{ Stress } \\
\hline Light & 5135 & 60 & 1.17 & 1 & & \\
\hline Heavy & 2159 & 27 & 1.25 & 1.097 & $0.623-1.934$ & 0.7483 \\
\hline \multicolumn{7}{|c|}{ Influenza vaccination } \\
\hline$(-)$ & 4806 & 55 & 1.14 & 1 & & \\
\hline$(+)$ & 2515 & 32 & 1.27 & 1.059 & $0.554-2.022$ & 0.8630 \\
\hline \multicolumn{7}{|l|}{ Hypertension } \\
\hline$(-)$ & 5847 & 73 & 1.25 & 1 & & \\
\hline$(+)$ & 1496 & 15 & 1.00 & 0.651 & $0.288-1.471$ & 0.3020 \\
\hline \multicolumn{7}{|l|}{ Diabetes mellitus } \\
\hline$(-)$ & 6774 & 84 & 1.24 & 1 & & \\
\hline$(+)$ & 569 & 4 & 0.70 & 0.287 & $0.098-0.842$ & 0.0230 \\
\hline \multicolumn{7}{|l|}{ Bronchial asthma } \\
\hline$(-)$ & 7096 & 82 & 1.16 & 1 & & \\
\hline$(+)$ & 225 & 5 & 2.22 & 1.733 & $0.526-5.705$ & 0.3658 \\
\hline \multicolumn{7}{|l|}{ Allergic rhinitis } \\
\hline$(-)$ & 6587 & 67 & 1.02 & 1 & & \\
\hline$(+)$ & 734 & 20 & 2.72 & 3.862 & $2.183-6.832$ & $<0.0001$ \\
\hline \multicolumn{7}{|c|}{ Nasal septum deviation } \\
\hline$(-)$ & 4206 & 47 & 1.12 & 1 & & \\
\hline$(+)$ & 3125 & 41 & 1.31 & 1.091 & $0.626-1.902$ & 0.7578 \\
\hline
\end{tabular}

$\mathrm{CRS}=$ chronic rhinosinusitis $\mathrm{OR}=$ odds ratio $; \mathrm{Cl}=$ confidence interval.

Kim's studies was the same as CRS that was diagnosed when the symptoms of both nasal obstruction and nasal discharge lasted for 3 months or longer. At the same time, intranasal endoscopic examination was used to confirm objective findings such as discolored nasal drainage or nasal polyps ${ }^{(13,15)}$. Furthermore, the survey time was the same; the only difference was the age of subjects, as Cho's study included subjects older than 6 years and Kim's study included subjects older than 12 years. Thus, the prevalence in Cho's and Kim's studies was similar at 7.12\% and $6.95 \%$, respectively ${ }^{(13,15)}$. However, in Min's study, CRS was defined as the presence of at least three clinical symptoms lasting longer than 3 months along with physical findings of a nasal polyp and/or mucopurulent nasal discharge within the middle meatus by rigid endoscopy. Nasal obstruction, rhinorrhea, 
anosmia or hyposmia as well as frontal, maxillary and interorbital pressure or headache were considered to be symptoms due to CRS. In Min's study, the prevalence of CRS was $1.01 \%{ }^{(12)}$ The discrepancy between the prevalence of endoscopy-based CRS of this present study and the previous reports of Kim et al. and Cho et al. can be explained as follows. According to the KNHANES conducted in 2008, the endoscopy-based CRS was defined as presence of discolored nasal discharge in the nasal passage or nasal polyps, therefore discolored discharge in the nasal cavity was considered positive. However, this study on KNHANES conducted in 2009 defines the endoscopy-based CRS as presence of mucopurulent rhinorrhea in the middle meatus or nasal polyps, and therefore only the presence of these findings were strictly considered for positive findings, excluding mucopurulent rhinorrhea in nasal cavity. The fact that the prevalence of endoscopy-based CRS of this study is similar to that of the report made by Min et al. strongly suggests that the discrepancy is due to differences in the definition of endoscopic findings. In actual fact, this discrepancy between the prevalence of endoscopy-based CRS of this study and the previous reports of Kim et al. and Cho et al. suggests the importance of diagnostic criteria utilized for CRS.

In this study, we also evaluated the strength of agreement of symptom-based diagnosis with reference to endoscopy-based diagnosis. The strength of agreement was low and this could be due to the low prevalence of endoscopy-based CRS as a standard for CRS diagnosis. The PPV and NPV are the proportions of positive and negative results in statistics and diagnostic tests that are true positive and true negative results ${ }^{(16)}$. Compared to endoscopy-based CRS, the sensitivity of symptom-based CRS was 1 and the specificity was 0.903 , PPV was 0.110 , and NPV was 1. All subjects diagnosed with endoscopy-based CRS were included in the symptom-based CRS group, according to the definitions of CRS in this study. Therefore, the sensitivity and NPV of symptom-based CRS were estimated at 1. The PPV of symptom-based diagnosis was low due to the low prevalence of endoscopy-based CRS. Lange showed that comparing CRS diagnosed by a questionnaire to CRS diagnosed by an otolaryngologist exhibits moderate agreement ${ }^{(17)}$. Our results coincide with Lange's study.

In evaluating symptom-based diagnosis with the addition of nasal endoscopy, several reports have raised debates on whether endoscopy should be regarded as a golden standard or not. Two independent studies by Fergusson and Stankiewicz showed that mucopurulence in endoscopic findings was relatively insensitive for the diagnosis of CRS ${ }^{(18,19)}$. The sensitivity for the endoscopic diagnosis of CRS was, respectively, $24 \%$ in the Fergusson study and $46 \%$ in the Stankiewicz study. Nevertheless, several studies do support the reliability of EP3OS symptom criteria and nasal endoscopy in the assessment of CRS. Bhattacharyya mentioned that in patients who meet symptom criteria for CRS, the addition of nasal endoscopy improves the diagnostic accuracy for CRS, and should be emphasized as a diagnostic tool early in clinical evaluations ${ }^{(20)}$. Moreover, the GA2LEN study revealed a significant association between symptom-based CRS and positive endoscopic findings ${ }^{(14)}$. Thus, the specificity of symptom-based diagnosis can indeed be strengthened by the addition of nasal endoscopy, as Bhattacharya et al. mentioned ${ }^{(20)}$. The determination of inter-rater agreement is an important component of the validation process for any diagnostic tool. McCoul et al. investigated inter-rater agreement of nasal endoscopic examination and showed excellent endoscopic agreement for the assessment of polyps, middle turbinate integrity, middle turbinate position, and maxillary or ethmoid sinus patency. However, discharge, synechiae, middle meatus patency, and middle turbinate mucosal changes showed fair or poor agreement with endoscopic findings (21). In the present study, 'endoscopy-based CRS' was defined as a diagnosis based on endoscopic findings of mucopurulent rhinorrhea in the middle meatus or nasal polyps along with nasal symptoms. Therefore, our definition would be somewhat reliable in light of McCoul's study.

The prevalence of symptom-based CRS was higher in men and increased with age, heavy stress, allergic rhinitis and nasal septum deviation. The prevalence of endoscopy-based CRS was higher in men and increased with age; less education and allergic rhinitis also increased the prevalence. Many risk factors affected CRS prevalence in previous studies. Pilan's study showed that a low monthly income and history of asthma and rhinitis increased the prevalence of CRS in Brazil ${ }^{(6)}$. In Chen's study in Canada, CRS was more common among those with a history of allergies, asthma, or chronic obstructive pulmonary disease (11). In Shi's study in China, chronic sinusitis was particularly prevalent among people with specific medical conditions, including allergic rhinitis, asthma, chronic obstructive pulmonary disease, and gout ${ }^{222}$. Although many risk factors demonstrated an impact on the prevalence of CRS in previous studies, it is difficult to compare risk factors between other countries because of racial, climatic, and cultural differences. Considering this fact, we compared two studies performed in the same country and concluded allergic rhinitis was identified as a common risk factor for CRS based on the two diagnostic criteria. However, our diagnostic criteria of 'endoscopy-based CRS' would affect the prevalence of CRS, so the association between allergic rhinitis and CRS can't be certain. In Min's study, the prevalence of CRS was significantly associated with province, employment status, the number of living rooms per family (crowding) and allergic rhinitis symptoms ${ }^{(12)}$. Kim et al. showed that allergic rhinitis is the most significant risk factor for CRS at the population level in Korea, and our results support Kim et al.'s findings ${ }^{(13)}$. Moreover, several studies have shown that allergic rhinitis and CRS exhibit strong epidemiological co-association, and early life risk factors for upper airway disease are now apparent ${ }^{(23-25)}$. Although exact 
mechanisms remain unknown, the inflammatory effect of allergic rhinitis can exacerbate underlying CRS ${ }^{(26,27)}$. Excess mucosal inflammation with immune dysfunction is a common feature for both allergic rhinitis and CRS. The risk factors for CRS in Korea were quite different except for allergic rhinitis, although all analyses were based on the nationwide survey. In these two studies, there was a time difference of 15 years and socioeconomic status varied considerably. Furthermore, the prevalence of allergic rhinitis considerably increased in 2011 , and this increased prevalence of allergic rhinitis can explain the increased prevalence of CRS despite the different diagnostic criteria for CRS ${ }^{(14)}$.

\section{Conclusion}

In conclusion, the prevalence and risk factors of CRS were quite different from each other according to the different criteria, even in the same population. Therefore, it would be important to consider what specific diagnostic criteria have been adopted in the studies comparing the prevalence of CRS.

\section{Acknowledgements}

The authors thank Han Na Yoo, PhD, for statistical consultation for this study.

\section{Authorship contribution}

All authors had full access to all data in the study and take responsibility for the integrity of the data and accuracy of data analysis. JHK: wrote the paper as a primary author; CC: discussed study design and analyzed the results; EJL: analyzed the results and contributed to write the paper; YSS and BIC: organized the research data. KSK: conceived the study and supervised this study as the senior clinician.

\section{Conflict of interest}

The authors have declared that no competing interests exist.

\section{References}

1. Benninger MS, Ferguson BJ, Hadley JA, et al. Adult chronic rhinosinusitis: definitions, diagnosis, epidemiology, and pathophysiology. Otolaryngol Head Neck Surg. 2003 129: S1-S32.

2. Gliklich RE, Metson R. The health impact of chronic sinusitis in patients seeking oto laryngologic care. Otolaryngol Head Neck Surg. 1995; 113: 104-109.

3. Kaliner MA, Osguthorpe JD, Fireman P, et al Sinusitis: bench to bedside. Current findings, future directions. Otolaryngol Head Neck Surg. 1997; 116: S1-S20.

4. Van Agthoven M, Fokkens WJ, van de Merwe JP, Marijke van Bolhuis E, Uyl-de Groot CA, Busschbach JJ. Quality of life of patients with refractory chronic rhinosinusitis: effects of filgrastim treatment. Am J Rhinol. 2001; 15: 231-237.

5. Halawi AM, Smith SS, Chandra RK. Chronic rhinosinusitis: epidemiology and cost Allergy Asthma Proc. 2013; 34: 328-334.

6. Pilan RR, Pinna FR, Bezerra TF, et al Prevalence of chronic rhinosinusitis in Sao Paulo. Rhinology. 2012; 50: 129-138.

7. Hastan D, Fokkens WJ, Bachert C, et al. Chronic rhinosinusitis in Europe--an underestimated disease. A GA2LEN study. Allergy. 2011; 66: 1216-1223.

8. Lange $B$, Holst $R$, Thilsing $T$, Baelum J, Kjeldsen A. Quality of life and associated factors in persons with Chronic Rhinosinusitis in the general population. Clin Otolaryngol. 2013; 38: 474-480.

9. Fokkens WJ, Lund VJ, Mullol J, et al. European Position Paper on Rhinosinusitis and Nasal Polyps 2012. Rhinol Suppl. 2012; 3: 1-298.

10. Pleis JR, Ward BW, Lucas JW. Summary health statistics for U.S. adults: National Health Interview Survey, 2009. Vital Health Stat 10. 2010; 249: 1-207.

11. Chen $Y$, Dales R, Lin M. The epidemiolo- gy of chronic rhinosinusitis in Canadians Laryngoscope. 2003; 113: 1199-1205.

12. Min YG, Jung HW, Kim HS, Park SK, Yoo KY Prevalence and risk factors of chronic sinusitis in Korea: results of a nationwide survey. Eur Arch Otorhinolaryngol. 1996; 253: 435439

13. Kim YS, Kim NH, Seong SY, Kim KR, Lee GB, Kim KS. Prevalence and risk factors of chronic rhinosinusitis in Korea. Am J Rhinol Allergy. 2011; 25: 117-121.

14. Tomassen P, Newson RB, Hoffmans R, et al. Reliability of EP3OS symptom criteria and nasal endoscopy in the assessment of chronic rhinosinusitis--a GA ${ }^{2}$ LEN study. Allergy. 2011, 66: 556-561.

15. Cho YS, Choi SH, Park KH, et al. Prevalence of otolaryngologic diseases in South Korea: data from the Korea national health and nutrition examination survey 2008. Clin Exp Otorhinolaryngol. 2010; 3: 183-193.

16. Robert H. Fletcher, Suzanne W. Fletcher Clinical epidemiology : the essentials 4th ed. Baltimore: Lippincott Williams and Wilkins, 2005

17. Lange B, Thilsing T, Baelum J, Holst R, Kjeldsen A. Diagnosing chronic rhinosinusitis: comparing questionnaire-based and clinical-based diagnosis. Rhinology. 2013; 51: 128-136.

18. Ferguson BJ, Narita M, Yu VL, Wagener MM Gwaltney JM Jr. Prospective observational study of chronic rhinosinusitis: environmental triggers and antibiotic implications. Clin Infect Dis. 2012; 54: 62-68.

19. Stankiewicz JA, Chow JM. Nasal endoscopy and the definition and diagnosis of chronic rhinosinusitis. Otolaryngol Head Neck Surg. 2002: 126: 623-627.

20. Bhattacharyya N, Lee LN. Evaluating the diagnosis of chronic rhinosinusitis based on clinical guidelines and endoscopy. Otolaryngol Head Neck Surg. 2010; 143: 147-151.
21. McCoul ED, Smith TL, Mace JC, et al. Interrater agreement of nasal endoscopy in patients with a prior history of endoscopic sinus surgery. Int Forum Allergy Rhinol. 2012; 2: 453-459.

22. Shi JB, Fu QL, Zhang $H$, et al. Epidemiology of chronic rhinosinusitis: results from a cross-sectional survey in seven Chinese cities. Allergy. 2015; 70: 533-539.

23. Furukawa $\mathrm{CT}$. The role of allergy in sinusitis in children. J Allergy Clin Immunol. 1992; 90: 515-7.

24. Iwens P, Clement PA. Sinusitis in allergic patients. Rhinology. 1994; 32: 65-7.

25. Berrettini S, Carabelli A, Sellari-Franceschini $S$, et al. Perennial allergic rhinitis and chronic sinusitis: correlation with rhinologic risk factors. Allergy. $1999 ; 54$ :242-8.

26. Krouse $\mathrm{JH}$. Allergy and chronic rhinosinusitis. Otolaryngol Clin North Am. 2005; 38: 1257-66.

27. Ryan MW, Brooks EG. Rhinosinusitis and comorbidities. Curr Allergy Asthma Rep. 2010; 10: 188-93.

Kyung-Su Kim, MD, PhD

Department of Otorhinolaryngology

Gangnam Severance Hospital

Yonsei University College of Medi-

cine,

211 Eonju-ro, Gangnam-gu,

135-720 Seoul

Republic of Korea

Tel: +82220193460

Fax: +82234634750

E-mail:ydrhinol@yuhs.ac 